\title{
De novo transcriptome of Ischnura elegans provides insights into sensory biology, colour and vision genes
}

Pallavi Chauhan ${ }^{1 *}$, Bengt Hansson ${ }^{1}$, Ken Kraaijeveld ${ }^{2,3}$, Peter de Knijff ${ }^{3}$, Erik I Svensson ${ }^{1}$ and Maren Wellenreuther ${ }^{1 *}$

\begin{abstract}
Background: There is growing interest in odonates (damselflies and dragonflies) as model organisms in ecology and evolutionary biology but the development of genomic resources has been slow. So far only one draft genome (Ladona fulva) and one transcriptome assembly (Enallagma hageni) have been published. Odonates have some of the most advanced visual systems among insects and several species are colour polymorphic, and genomic and transcriptomic data would allow studying the genomic architecture of these interesting traits and make detailed comparative studies between related species possible. Here, we present a comprehensive de novo transcriptome assembly for the blue-tailed damselfly Ischnura elegans (Odonata: Coenagrionidae) built from short-read RNA-seq data. The transcriptome analysis in this paper provides a first step towards identifying genes and pathways underlying the visual and colour systems in this insect group.

Results: Illumina RNA sequencing performed on tissues from the head, thorax and abdomen generated $428,744,100$ paired-ends reads amounting to $110 \mathrm{~Gb}$ of sequence data, which was assembled de novo with Trinity. A transcriptome was produced after filtering and quality checking yielding a final set of 60,232 high quality transcripts for analysis. CEGMA software identified 247 out of 248 ultra-conserved core proteins as 'complete' in the transcriptome assembly, yielding a completeness of 99.6\%. BLASTX and InterProScan annotated 55\% of the assembled transcripts and showed that the three tissue types differed both qualitatively and quantitatively in I. elegans. Differential expression identified 8,625 transcripts to be differentially expressed in head, thorax and abdomen. Targeted analyses of vision and colour functional pathways identified the presence of four different opsin types and three pigmentation pathways. We also identified transcripts involved in temperature sensitivity, thermoregulation and olfaction. All these traits and their associated transcripts are of considerable ecological and evolutionary interest for this and other insect orders.

Conclusions: Our work presents a comprehensive transcriptome resource for the ancient insect order Odonata and provides insight into their biology and physiology. The transcriptomic resource can provide a foundation for future investigations into this diverse group, including the evolution of colour, vision, olfaction and thermal adaptation.
\end{abstract}

Keywords: Odonata, Zygoptera, Polymorphisms, Transcriptome assembly, RNA-seq, Opsin, Melanin, Ommochrome and pteridine, Thermal adaptation

\footnotetext{
*Correspondence: pallavi.chauhan@biol.lu.se; maren.wellenreuther@biol.lu.se 'Department of Biology, Lund University, Sölvegatan 37, SE-22362 Lund, Sweden Full list of author information is available at the end of the article
} 


\section{Background}

Odonata display large inter- and intra-specific colour variation and have some of the most advanced visual systems among insects [1,2]. With their large and complex eyes, aquatic and terrestrial life stages [3], carnivorous lifestyle [4], exceptional mating behaviours [5,6], diversity in coloration [7], and occupancy of diverse light environments, odonates are ideal model organisms to study the evolution of colour and vision pathways and functions. However, odonate colour and visual systems are little understood [8]. Lack of genomic and transcriptomic sequence information limits molecular investigation on this group. So far only one draft genome (BioProject PRJNA194433, Ladona fulva) and one transcriptome assembly [9] have been published for odonates. An improved understanding of the molecular basis of phenotypic adaptations in Odonata would allow investigations of genomic divergence associated with ecological shifts in light environments, and inter- and intra-specific divergence in color vision. Several distinctive traits of the blue-tailed damselfly Ischnura elegans (Odonata: Coenagrionidae) make this species a useful model for studying genome evolution and development. Ischnura elegans has developed into a model organism in evolutionary ecology because of its female limited colour polymorphism, which affects mate choice and sexual conflict interactions. Males of I. elegans are monomorphic in colour, but females of this species fall into one of three distinct phenotypically visible colour morphs, namely the male mimicking androchrome morph, and the more cryptic infuscans and infuscans-obsoleta morph [10]. The prevalence of female colour polymorphism in this species is thought to result from sexual conflict over optimal mating rates, where females might benefit from lower mating rates than males, and where pre-copulatory male mating harassment is common [11,12]. This sexual conflict leads to extensive mating harassment and negative frequency-dependent selection, because the males form search images for the common morphs, similar to the apostatic survival selection on common prey caused by predators [13]. This species has also been studied with respect to sperm competition $[14,15]$, morph dependent mating rates $[11,12]$, and the evolution of reproductive barriers [16,17]. Ischnura elegans belongs to the largest damselfly family Coenagrionidae, which includes 95 genera and 1082 species worldwide [18]. Over 100 species are colour polymorphic [19], and evidence from crossing experiments in several species suggests a genetic basis to colour [20,21]. In the female-polymorphic genus Ischnura, even closely related taxa often differ in the presence and absence of female polymorphism and/or in the spectral ability to differentiate colour [22]. Identifying the genetic changes underlying the colour polymorphism on an intra- and inter-specific level would increase our understanding of the macroevolutionary dynamics of this polymorphism. Ischnura elegans is a widespread damselfly species all over Europe [23,24] and can commonly be found in disturbed environments, such as human-made artificial ponds [13]. Unlike many other odonate species, I. elegans tolerates most plants as perching substrate [3].

Here we present a de novo transcriptome assembly for the blue-tailed damselfly I. elegans to investigate the nuclear, protein-encoding gene profile of this species and to give functional annotation to the proteins expressed. The transcriptome of the head, thorax and abdomen are compared to each other, and to the transcriptome of the dragonfly Ladona fulva (Odonata: Anisoptera) [BCMHGSC:I5K] [25], the damselfly Enallagma hageni (Odonata: Zygoptera) [NCBI:SRR649536] $[9,26]$ and the fruit fly Drosophila melanogaster (Diptera) [Ensembl:BDGP5] [27]. Furthermore, we aim to generate a sensory toolkit for the genes underlying colour recognition (e.g. opsins), female polychromatism and body colour patterns (e.g. melanin pathway).

\section{Results and discussion}

\section{Transcriptome sequencing}

Illumina sequencing of one I. elegans individual yielded a total of $110 \mathrm{~Gb}$ of mRNA sequence equivalents consisting of $428,744,100$ paired-ends 100 bp reads $(155,232,504$ reads from the head, 159,734,116 from the thorax and $113,777,480$ from the abdomen, respectively). The average read length for each of the three tissues was $99 \mathrm{bp}$, yielding complete datasets of $39.8 \mathrm{~Gb}$ for the head, $40.8 \mathrm{~Gb}$ thorax and $29.2 \mathrm{~Gb}$ for the abdomen. Quality parameters of the three tissues types (head, thorax and abdomen) were $91 \%, 92 \%$, 92\% for Q20, and 42\%, 38\%, and 43\% for the GC percentage, respectively, while the percentage of unknown base calls $(\mathrm{N})$ was $0.007 \%$ for both the head and thorax and $0.005 \%$ for the abdomen.

High quality data (clean reads) were obtained by removing reads containing adapter sequences, short reads and low quality reads from raw sequence data, reducing the three sequence sets to approximately 129, 134, and 95 million reads for the head, thorax and abdomen, respectively. Subsequently, the trimmed reads from the head, thorax and abdomen had a mean read length of 83, 84 and $82 \mathrm{bp}$, respectively, and the Q20 percentage was $100 \%$ for all three tissues. For detail summary of the trimming step statistics refer to Table 1 . All the subsequent analyses were carried out using 357,641,660 high quality trimmed reads.

\section{De novo transcriptome assembly, quality filtering and assessment}

The transcriptome was assembled de novo with Trinity $[28,29]$ using all trimmed reads and yielded a total of 89,708 contigs with a minimum length of 201 bp, a N50 
Table 1 Trimming report

\begin{tabular}{llll}
\hline & Head & Thorax & Abdomen \\
\hline Number of reads before trimming & 155232504 & 159734116 & 113777480 \\
Reads kept after trimming & 128679780 & 133566318 & 95395562 \\
$\begin{array}{l}\text { Percentage of reads discarded } \\
\text { Reads average length }\end{array}$ & $17.1 \%$ & $16.4 \%$ & $16.2 \%$ \\
$\begin{array}{l}\text { before trimming } \\
\text { Reads average length }\end{array}$ & 99 & 99 & 99 \\
after trimming & 83 & 84 & 82 \\
$\begin{array}{l}\text { Q20\% before trimming } \\
\text { Q20\% after trimming }\end{array}$ & $91 \%$ & $92 \%$ & $92 \%$ \\
$\begin{array}{l}\text { Q30\% before trimming } \\
\text { Q30\% after trimming }\end{array}$ & $100 \%$ & $100 \%$ & $100 \%$ \\
Total high quality reads & $83 \%$ & $84 \%$ & $83 \%$ \\
\hline
\end{tabular}

value of 2,610 bp and an average contig length of 1,213 bp. In the absence of a reference genome it is difficult to assess the quality of the assembled transcripts. However, to identify poor quality and potentially mis-assembled transcripts, the reads were mapped back to the assembly and the alignment visualized with IGV v.2.3.2 [30].

To improve the overall quality of the assembled transcriptome, a three-step quality filtering method was employed [31-33]. First, sequence redundancy was removed by clustering the duplicates using CDHIT-EST at 95\% sequence similarity [34]. This step clustered 14.8\% of the transcripts together, leaving 76,356 transcripts. Second, the transcript read coverage at each base was calculated using BED Tools. Transcripts that had a mean coverage per base of less than 5 were removed, filtering 16,008 transcripts, and leaving 60,348 high quality transcripts. Third, RepeatMasker [35] identified 17,467 repetitive elements, 178 RNA (tRNA, rRNA and srpRNA) and 2,691 low complexity regions in the transcriptome (Additional file 1: Table S1). From this, 138 ribosomal RNA sequences were identified in 116 transcripts $(0.2 \%)$ and these were removed from the assembly. The filtering step not only reduced the redundancy but also filtered the shorter sequences (Additional file 2: Figure S1). Comparative assembly statistics before and after filtering are reported in Additional file 1: Table S2. The final dataset contained 60,232 high quality transcripts with an N50 value of 2,571 bp and a mean length of 1,281 bp, which was used for all subsequent analyses. A detailed summary of the final assembly statistics can be found in Table 2.

After quality filtering, the assembly was further validated for sequence completeness. CEGMA [36] identified 247 out of 248 ultra-conserved core proteins as 'complete' in the transcriptome assembly, yielding a completeness of $99.6 \%$. The remaining gene was identified as a 'partial' gene. TargetIdentifier [37] identified
Table 2 Summary statistics of final assembly

\begin{tabular}{ll}
\hline Assembly assessment parameters & Final transcript set \\
\hline Number of contigs & 60232 \\
Total size of contigs (bp) & 77140699 \\
Longest contig (bp) & 24097 \\
Shortest contig (bp) & 201 \\
Mean contig size (bp) & 1281 \\
Median contig size (bp) & 627 \\
N50 contig length (bp) & 2571 \\
Number of contig > 500 nt & 34746 \\
Number of contig > 1000 nt & 22164 \\
\hline
\end{tabular}

23,021 transcripts with a BLASTX hit, of which 15,949 transcripts (69\%) could be assembled to their full length. Of these transcripts, 14,301 were identified as fulllength, 1,496 as short full-length, 152 as ambiguous, 3,983 as $5^{\prime}$-sequenced partial and 3,089 as $3{ }^{\prime}$-sequenced partial. The full-length information was generated only for the transcripts that yielded a BLASTX hit. Further, the assembly was investigated for the ability to yield protein-coding sequences. TransDecoder reported 24,885 ORFs in 21,317 (35.4\%) transcripts. The assembly sequence completeness and protein-yielding capability was high, and hence the assembly was used for further analysis.

\section{Transcript annotation}

The transcript annotation with BLASTX [38] using the NCBI non-redundant $(\mathrm{nr})$ protein database gave a match for 22,995 (38.2\%) transcripts, while 37,237 transcripts could not be matched with BLASTX using a cut-off of $1 \mathrm{E}-5$. The species representing the top BLAST hits are shown in Figure 1A. Of these, Tribolium castaneum (Coleoptera) yielded the highest number of top hits, followed by Pediculus humanus (Phthiraptera) and Megachile rotundata (Hymenoptera). All three orders belong to the Neoptera reviewed in [39], which is considered the closest relative to the Odonata (Metapterygota). Of the three orders, the Phthiraptera are taxonomically closest to the Odonata, followed by the Hymenoptera and then the Coleoptera [39,40], which was not reflected in our BLASTX homology search. The small genome size of Phthiraptera and its high evolutionary rate (long branch length [40]), likely due to the parasitic lifestyle, possibly partly explains the lower number of hits in comparison to Coleoptera. Moreover, all the three orders diverged from Odonates a long time ago and during a relatively small time window, making a distinction between them difficult. Lastly, the homology results will likely be biased by the amount and quality of available species-specific data that has been deposited in the databases. Closely related species with genomic resources were compared to the 


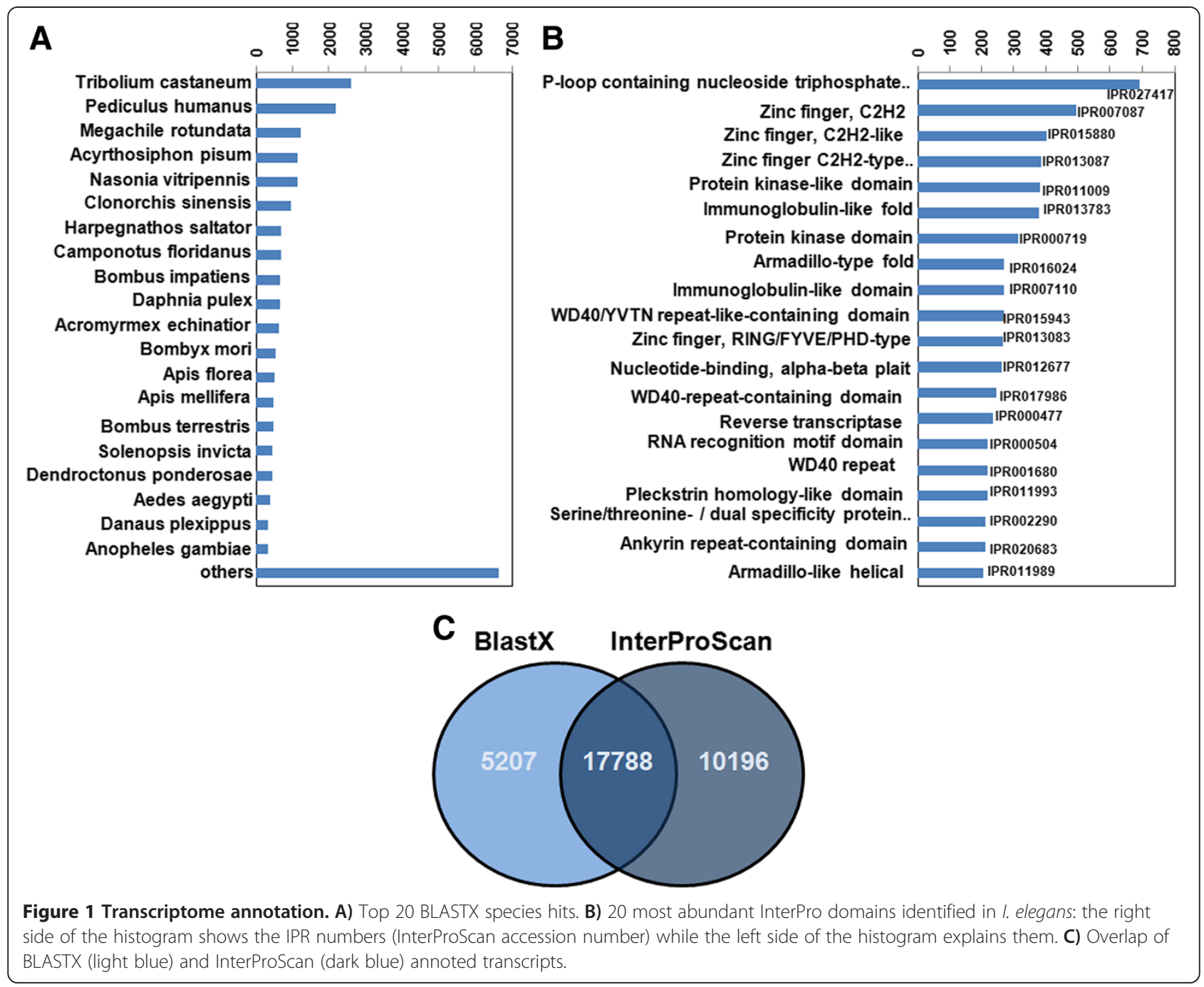

I. elegans transcriptome by mapping our data to the transcriptome of the dragonfly species Ladona fulva (Odonata), the damselfly species Enallagma hageni (Odonata) and the fruit fly model Drosophila melanogaster (Diptera).

The BLAST2GOInterProScan annotation resulted in 27,984 transcripts (46.5\%) with at least one InterProScan annotation. A list of the 20 most abundant InterPro domain hits is reported in the Figure 1B, showing IPR027417 (P-loop containing nucleoside triphosphate hydrolase) to be the most prevalent domain present in 691 transcripts, followed by IPR007087 (Zinc finger, $\mathrm{C} 2 \mathrm{H} 2$ ) and IPR015880 (Zinc finger, C2H2 like). The assembled transcripts were also annotated with Gene Ontology (GO) into three major GO categories: Biological Processes, Cell Component, and Molecular Function. A total of 11,748 (20\%) transcripts were associated with at least one GO term: 6,393 transcripts were assigned to the Biological Processes, 10,483 to the Molecular Function and 3,848 to Cell Components (Additional file 2: Figures S2, S3 and S4). In the Biological Process category, the majority of transcripts were involved in cellular protein metabolic processes (GO: 0006464) and signal transduction processes (GO: 0007165). A large fraction of transcripts in the Molecular Function category is involved in DNA binding (GO: 0003677) and RNA binding (GO: 0003723) functions, whereas the Cellular Component category is predominated by transcripts involved in intracellular organelle (GO: 0043229) and cytoplasm (GO: 0005737) processes.

BLASTX was able to annotate $38.2 \%$ and InterProScan $46.5 \%$ of the transcripts. Considering both the BLASTX and InterProScan results, a total of 33,191 (55.1\%) de novo assembled transcripts could be annotated. Of the total number of annotated transcripts, 17,788 transcripts were well annotated (assigned with a gene name as well 
as a protein signature), obtained from union of BLASTX and InterProScan annotated transcripts, detailed in Figure $1 \mathrm{C}$.

\section{RNA-sequence mapping on the $D$. melanogaster and I. elegans transcriptome}

Almost all I. elegans trimmed sequence reads (99.4\% from all tissues) failed to map to the D. melanogaster transcriptome, which consists of 27,142 transcripts [27]. Of the $2,283,100$ reads $(0.6 \%)$ that mapped to the $D$. melanogaster transcriptome, 721,518 reads $(0.2 \%)$ were paired, whereas $1,561,582$ reads $(0.4 \%)$ mapped as singletons. Only $1,626(6.0 \%)$ of the $D$. melanogaster transcripts showed expression in I. elegans (a list of the 30 most expressed transcripts are shown in Additional file 1: Table S3).

RNA-seq reads from the dragonfly L. fulva [25] and the damselfly E. hageni [26] were mapped to the I. elegans transcriptome. The majority of reads $(88.9 \%)$ from the $L$. fulva could not be mapped to the I. elegans transcriptome. Among the 8,569,657 L. fulva reads that mapped to the $I$. elegans transcriptome, 4,565,885 (5.9\%) mapped as singletons and 4,003,772 (5.2\%) reads as pairs, generating an overall mapping percentage of $11.1 \%$. Mapping of $E$. hageni single-end reads to the $I$. elegans transcriptome resulted in 513,478 (52.6\%) reads that could not be mapped and 463,268 (47.4\%) of reads mapped. Summary statistics of RNA-seq reads for L. fulva and E. hageni mapping to the I. elegans transcriptome are reported in Table 3. More E. hageni (11.6\%) transcripts were found in I. elegans compared to L. fulva (3.7\%). Of these, 1,325 (2.2\%) transcripts were expressed in both the species (Additional file 2: Figure S5). They are presumably expressed in all three species, but homology is too low to detect most of them. A list of the 30 most expressed genes from both species is shown in Additional file 1: Table S4.

Interspecific transcript level comparisons showed that E. hageni shared most transcripts $(6,989)$ with I. elegans, followed by L. fulva $(2,244)$ and D. melanogaster $(1,626)$, which closely corresponds to the taxonomic distance between these species $[39,40]$. A large fraction of these common transcripts encodes for proteins that are involved in maintaining structure and function of muscles

Table 3 Statistics for RNA-seq mapping on I. elegans transcriptome

\begin{tabular}{lll}
\hline Mapping parameters & Ladona fulva & Enallagma hageni \\
\hline Total reads & 77135056 & 976765 \\
Reads mapped in pairs & 4003772 & - \\
Reads mapped in broken pairs & 4565885 & 463268 \\
Percentage of mapped reads & $11.11 \%$ & $47.43 \%$ \\
Reads not mapped & 68565399 & 513497 \\
\hline
\end{tabular}

and the transfer of electrons in the electron transport chain in mitochondria.

\section{Abundance estimation and differential expression of transcripts in the three tissue types}

RNA-seq mapping was performed with RSEM [41] to calculate expression levels of the assembled transcripts for the three tissue types. Mapping results showed that $54,856,781(85.3 \%)$ paired reads from the head, $55,385,379$ (83.0\%) from the thorax and 39,821,676 (83.5\%) from the abdomen mapped to the assembled transcript, resulting in more than $15 \%$ reads that could not be mapped to the final set of assembled transcripts. The RNA-seq mapping analysis revealed that more transcripts were expressed in the abdomen tissue than in the other two tissue types. The total number of transcripts expressed was $51,006(84.7 \%)$ in the head, 47,544 (79.0\%) in the thorax and 53,286 (88.5\%) in the abdomen, respectively. Comparative analyses among all the three tissues revealed that 41,866 (69.5\%) transcripts were expressed in all tissues, while 3,495 (5.8\%) of the transcripts were mutually expressed in the head and thorax, 3,937 (6.5\%) in the head and abdomen and lastly $1,661(2.8 \%)$ transcripts in the abdomen and thorax (Figure 2A).

A list of the 20 most expressed genes in the head, thorax and abdomen, respectively is reported in Figure 2B (extracted from Additional file 1: Tables S5, S6 and S7). Of the top 20 genes expressed in I. elegans tissues, the largest fraction was made up of proteins that act as components in the respiratory chain of mitochondria facilitating electron transfer (e.g. cytochrome b, cytochrome c, cytochrome oxidase and NADH dehydrogenase) and muscle proteins (e.g. muscle actin, muscle lib protein and myofilin). A hypothetical protein that showed highest expression in all the three tissue is an immune-related gene, which regulates immune signalling in insects [42]. Noteworthy, high expression of protein coding gene takeout [Flybase: FBgn0039298] was observed in head. This protein participates in a novel circadian output pathway and is also involved in male courtship behaviour in $D$. melanogaster.

A total of 8,625 transcripts were differentially expressed in the head (2,039), thorax (963) and abdomen $(5,623)$ using a p-value cut-off for FDR $1 \mathrm{E}-3$ and a fold change of 2 . However without biological replicates, any conclusions about gene expression data can be weak. A list of the 20 most differentially expressed genes in the head, thorax and abdomen, respectively, are reported in Figure 3A-C (based on data in Additional file 1: Tables S8-S10). The results clearly showed that the most differentially expressed genes in the head were opsins (longwavelength-sensitive 1, long-wavelength-sensitive 2 and blue-sensitive opsin), genes that participate in light- 


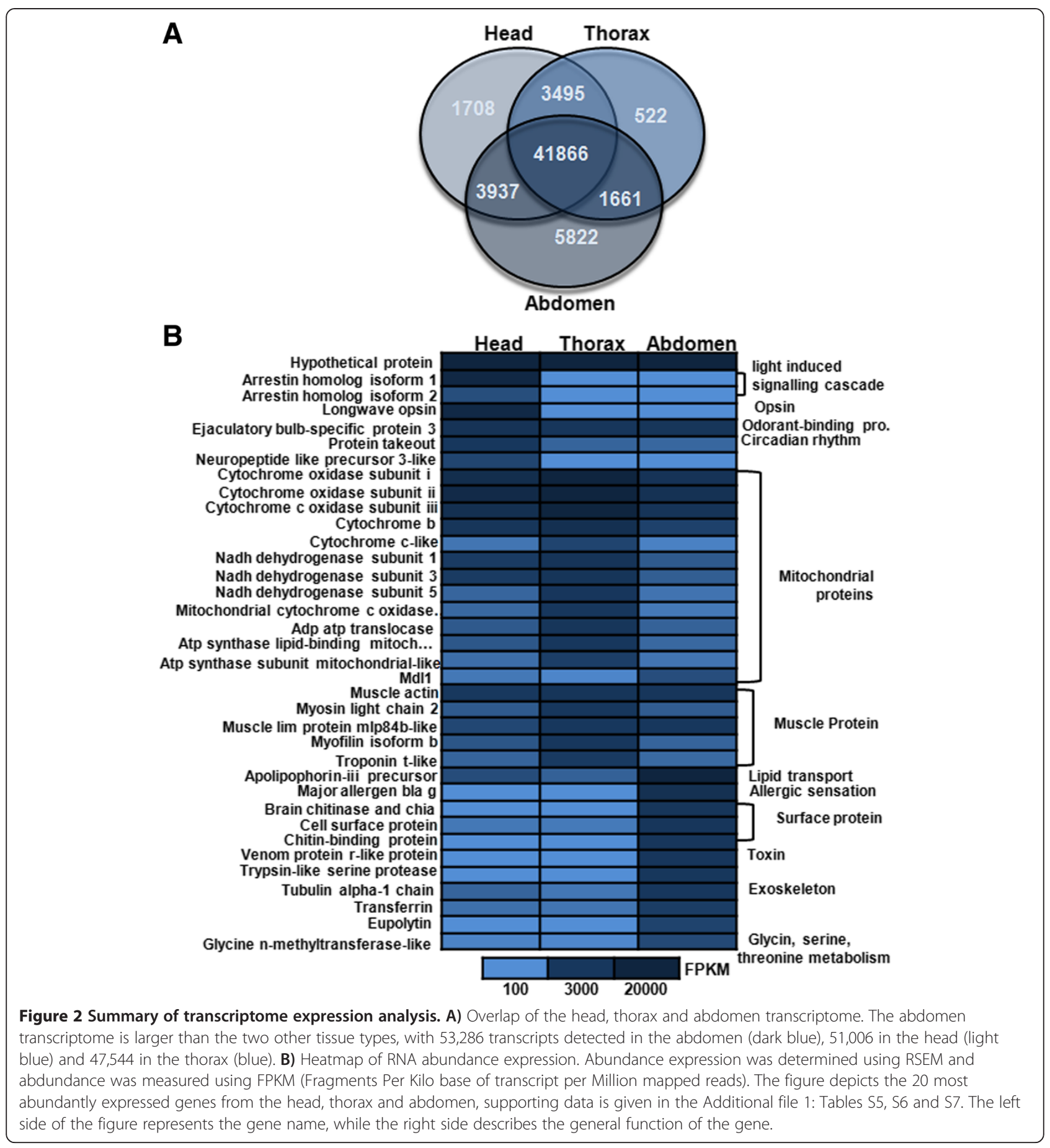

induced cascades (e.g. arrestin homolog isoform 1 and 2), and neuropeptide-like precursors that influence behaviour, development, immunity and physiological processes. The thorax showed elevated expression of genes that encode mitochondrial proteins. The differentially expressed genes of the abdomen included major allergen $B l a$, which is an allergen that is responsible for respiratory disorders like asthma [43], brain chitinase and chia, chitinase, chitin-binding protein, all these have chitin- binding domain and form peritrophic matrix proteins of insects, venom protein r-like, which is a toxin and hemolymph juvenile hormone binding protein, which regulates embryogenesis, larva development and stimulates reproductive maturation.

A GO term enrichment analysis was performed on the differentially expressed genes from the head, thorax and abdomen using GOSSIP [44] Fishers Exact Test with BLAST2GO. A total of 39 enriched GO terms were 


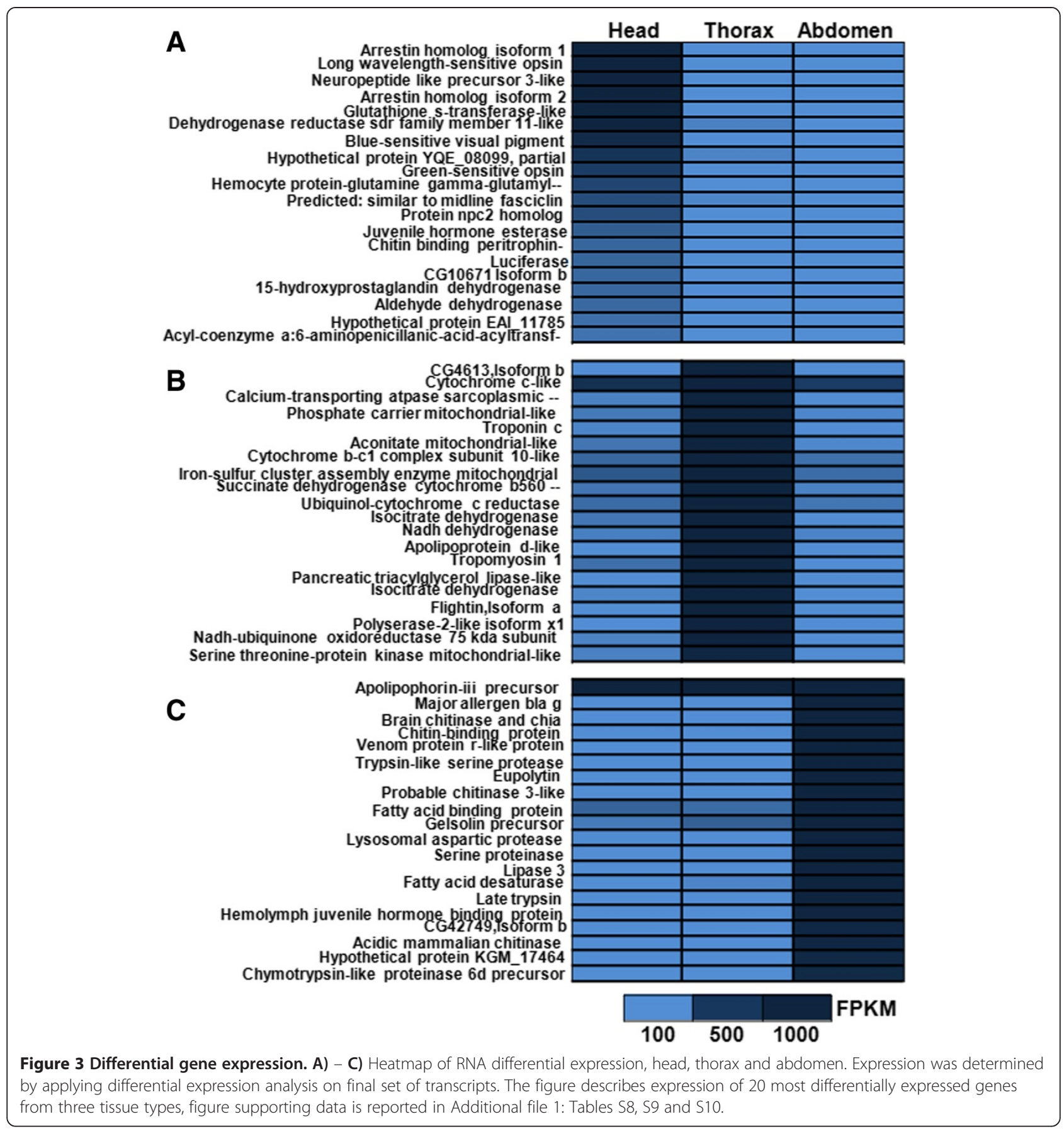

identified in the head, which were subsequently reduced to 11 most specific terms. The most specific GO terms in the head included signal transduction (GO: 0007165) and responses to abiotic stimuli (GO: 0009628) under the Biological Processes category, plasma membrane (GO: 0005886) and cytoplasmic membrane-bound vesicles (GO: 00016023) under the Cell Component category and receptor activity (GO: 0004872) and ion channel activity (GO: 0005216) under the Molecular Function category. Only three enriched GO terms were observed in the thorax, which included the generation of precursor metabolites and energy (GO: 0006091) under the Biological Processes category, mitochondrion (GO: 0005739) under Cell Component category and electron transport activity (GO: 0009055) under Molecular Function category. A total of 31 enriched GO terms were observed in the abdomen, of which 15 were reduced to the most specific GO terms. Among these, the most enriched GO terms were catabolic processes (0009056) and translation (GO: 0006412) under the Biological Processes category, 
ribosome (GO: 0005840) and cytoskeleton (GO: 0005856) functions under the Cell Component category and structural molecular activity (GO: 0005193) and peptidase activity (GO: 0008233) under the Molecular Function category (for details refer to the Additional file 2: Figures S6-S8).

The consistent findings from the abundance estimation and differential expression analysis underscore the specific roles that these three tissue types play in I. elegans: the head seems to regulate not only light receptivity and vision but also other sensory processes and transmits information via electrical and chemical signals to other body parts, response to abiotic stimulus and also contain protein that can regulate male courtship behaviour; the thorax with its flight musculature has a large number of mitochondria and muscle proteins and the abdomen not only performs translation and catabolic processes but also contains some defence proteins, such as allergens and toxins.

\section{Opsin and pigment pathways}

The odonate eye can detect colour from the ultraviolet (UV) $(\sim 300 \mathrm{~nm})$ to the long wavelength (LW) $(\sim 700 \mathrm{~nm})$ portion of the visible spectrum and is capable of discriminating polarized light $[2,45]$. Past studies of electrophysiology have demonstrated that odonates have between 3-5 opsin copies for colour detection (reviewed in 1). We identified four types of opsins (long-wavelength-sensitive opsin 1, long-wavelength-sensitive opsin 2, blue-sensitive opsin and ultraviolet-sensitive opsin) in 20 different transcripts. The BLASTX homology search revealed that, on average, the identified opsins have more than $60 \%$ similarity with the identified homologous protein, although several showed more than $80 \%$ similarity. The abundance estimation performed on all opsins reported a FPKM (Fragments Per Kilobase of transcript per Million mapped reads) value of at least 300 in the head tissue (Additional file 1: Table S11). Among the four types of opsins detected in I. elegans, the long-wavelength-sensitive opsin 1 showed the highest expression in the head tissue with a FPKM of 37,939 , followed by blue-sensitive opsin with a FPKM of 1,377 (Figure 4).

In pigmentation pathways, effector genes encode for cofactors and enzymes involved in the synthesis of pigments and patterning genes regulate the distribution of pigments by influencing the activating of effector gene [46,47]. We identified 12 enzymes in the 23 assembled transcripts belonging to three pigment pathways, namely the melanin (4), pteridine pathway (4) and ommochrome (4). The diagrammatic representation of the enzymes identified in I. elegans in all the three corresponding pathways is shown in Figure 5A-C. We also identified seven regulatory proteins (patterning genes) in ten transcripts.

The melanin pathway is of principal interest in insects because different components in the enzymatic pathway

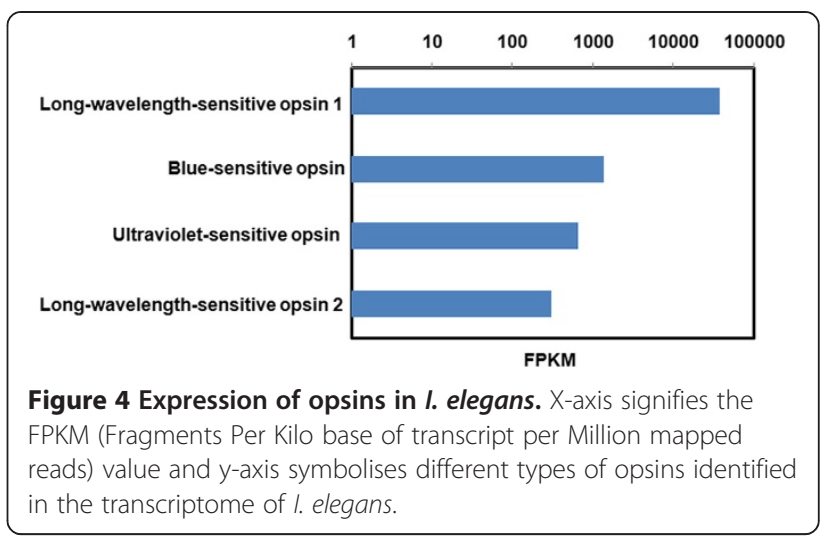

have different and pleiotropic effects on characters involved in mate choice, sexual selection and parasite resistance $[48,49]$ and possibly also learned mate preferences, which are known to occur in damselflies [50]. In the model insect $D$. melanogaster, dopamine is involved in the reward system and in learning, courtship and sexual behaviour [51-55]. The enzymes identified in $I$. elegans in the melanin pathway are tyrosine hydroxylase, dopa decarboxylase, yellow and phenoloxidases subunit a3 like, which all play important roles in forming black and brown colour pigments in other insects $[46,48,56-58]$. The phenoloxidase subunit a3 like is a copper containing oxidase that catalyses the rate-limiting conversion of tyrosine to DOPA, and DOPA to DOPA quinines $[59,60]$. In calopterygid damselflies, phenoloxidase is a limiting resource in terms of the life-history allocation between sexual signalling (dark wing patches in males) and innate immune defence against parasitic infections [50]. The ommochrome pathway yields red, brown and yellow pigments. The enzymes identified in I. elegans in the ommochrome pathway are tryptophan 2,3-dioxygenase like, kynurenine formamidase and kynurenine 3 monooxygenase present in cell cytosol $[61,62]$. The major facilitator superfamily plays an important role in the transport of 3-hydroxykynurenine into the pigment granules, where it undergoes oxidative condensation to form pigment xathommatin and ommins $[63,64]$. The pteridine biosynthesis pathway produces sepiapterin and biopterin, which are yellow and blue colours. The important enzymes identified in $I$. elegans in the pathway are guanosine triphosphate cyclohydrolase, sepiapterin reductase-like, dihydrofolate reductase, pterin-4a-carbinolamine dehydratase-like [65-67]. We identified all the enzymes involved in melanin, ommochrome and pteridine pathway in $I$. elegans, except one enzyme (pyruvoyl tetrahydropterine synthase, which converts dihydroneopterine triphosphate to 6-pyruvoyl tetrahydropterine) in the pteridine pathway. The BLASTX analysis performed on all enzymes showed an average similarity of $60 \%$ with the identified homologous protein. A detailed list of all the enzymes involved in 


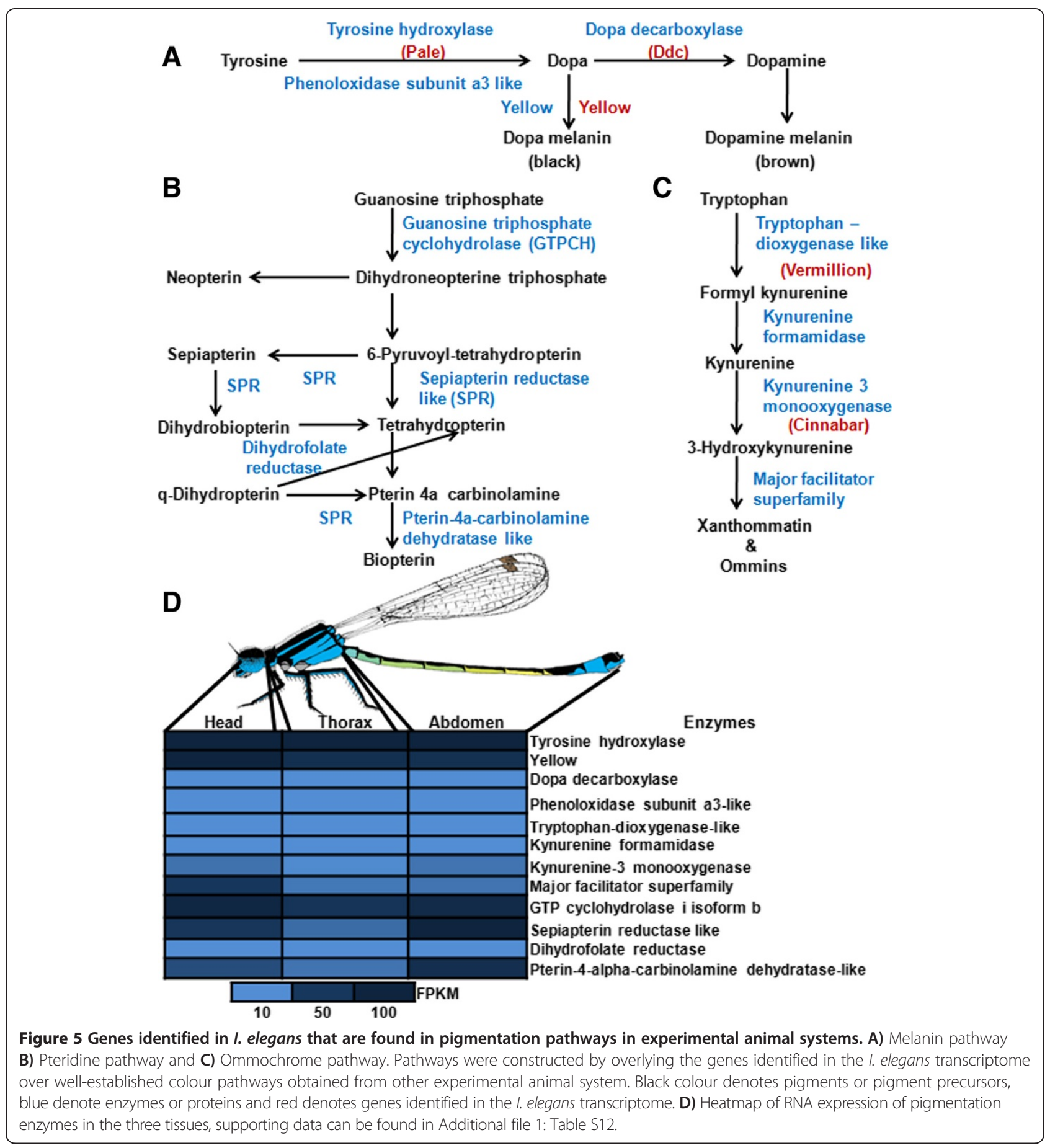

the three colour pathways with their expression in head, thorax and abdomen is shown in Figure 5D and Additional file 1: Table S12.

On the basis of RNA expression of the pigmentation enzymes reported in the Additional file 1: Table S12 and Figure 5D, the expression of tyrosine hydroxylase $(\mathrm{FPKM}=155)$ and yellow gene $(\mathrm{FPKM}=45)$ that both form black melanin is higher than dopa decarboxylase $(F P K M=1)$ that forms brown melanin in the head. This indicates the prominence of black melanin over brown melanin. A similar trend was also observed in the thorax (FPKM 203, 42 and $<1$ ) and abdomen $(185,44$ and $<1$ ). It hence appears that in I. elegans the formation of black melanin is prominent over brown melanin.

We were also able to identify a large number of patterning genes. We found seven regulatory genes in nine transcripts, namely doublesex, abdominal-a, wingless, decapentapleic, bric-a-brac, ultrabithorax and distal-less 
(Additional file 1: Table S12). Extensive pigmentation studies performed on D. melanogaster and other insects have reported a role of some of these regulatory elements in pigmentation patterning with strong links to sexual selection, sexual dimorphism and speciation in these more modern insect groups $[46,68,69]$. In the future, identification of pattering genes can help to answer questions related to sex-specific pigmentation in I. elegans and other odonate species that show genetic colour polymorphisms.

\section{Other important findings \\ Odorant-binding protein and receptors}

We identified odorant-binding proteins (ejaculatory bulb-specific protein 3 and odorant-binding proteins 4), Olfactory Receptor (OR), Ionotropic Receptors (IRs) and Gustatory Receptors (GRs) that were expressed in all three tissues (Additional file 1: Table S13). This is of principal interest as odonates have until quite recently been thought to have poor olfaction and mainly communicate through visual signals. Recent behavioural and electrophysiological work, however, indicates that olfaction might be important also in odonates, at least in the context of foraging [70,71]. Future studies on odonates should investigate if the odour and taste receptors that have been shown to be important in the detection of food, mates and oviposition sites in modern insects like Drosophila [72] are operating also in this very ancient insect group, which its long history of independent evolution from the well-investigated model insect systems.

\section{Heat and cold shock proteins}

We identified seven different types of heat shock proteins (HSP), three types of heat shock factors and two cold shock proteins in 35 transcripts. The HSP identified are HSP 10, HSP 70 (heat shock $70 \mathrm{kda}$ protein 14, heat shock $70 \mathrm{kda}$ protein $4 \mathrm{l}$, heat shock $70 \mathrm{kda}$ protein cognate 3 , heat shock protein $70 \mathrm{kda}$ protein cognate 5), HSP 75, HSP 60, HSP67b2, HSP90, HSPgp96, heat shock factor, heat shock factor 2-binding protein, heat shock factor binding and small HSP. The most expressed HSP in the head was HSP $70($ FPKM = 372) followed by HSP $90($ FPKM = 324), in the thorax small HSP $($ FPKM $=547)$ followed by HSP 70 $(\mathrm{FPKM}=173)$ and in the abdomen HSP $70(\mathrm{FPKM}=407)$ followed by smallHSP (FPKM = 239).

The cold shock proteins identified are cold shock domain-containing protein e1 and cold shock domain protein a. For detailed description about the expression and homology of heat and cold shock proteins refer Additional file 1: Table S14.

\section{Transient receptor potential (TRP) channel}

TRP channels are of ecological interest for research on thermal adaptation, as these insects are known to thermoregulate, in spite of being ectothermic animals $[73,74]$. Moreover, some of the larger odonates (dragonflies) can even generate heat internally, through muscle movement and thermogenesis [75]. The ability to thermoregulate is likely to be under strong natural and sexual selection, with latitudinal gradients and phylogenetic inertia are likely to have jointly shaped the phenotypic traits underlying thermal plasticity and thermal niches [7]. Here, we identified eight different types of TRP in 39 transcripts. The different types of TRP identified are TRP channel, TRP cation channel subfamily A member 1-like (TRPA1 isoform g, TRPA1 isoform $k$, TRPA1 isoform i), TRP cation channel subfamily v member 6 , TRP channel pyrexia, TRP cation channel protein painless-like, TRP cation channel cg34123-like, short TRP channel 5-like and TRP-gamma. In head TRP channelwas most expressed (FPKM $=98)$, followed by TRP cation channel subfamily A (FPKM = 28), whereas in thorax and abdomen TRP channel was most expressed (FPKM = 14 and FPKM =40), followed by TRP cation channel cg34123 (FPKM = 13 and FPKM = 32) (Additional file 1: Table S15).

\section{Conclusions}

The de novo transcriptome of $I$. elegans is the most complete transcriptome assembly of an odonate species to date and fills a major taxonomic gap. The annotated genes provide an important toolkit for future studies on colour, vision, olfaction and temperature sensitivity in this and other species. In particular, the data from this study will provide baseline knowledge for future studies investigating the molecular and genomic basis behind the evolution of colour polymorphism in Odonata, and the associated changes in vision, which may have facilitated phenotypic divergence in this ancient insect order. Moreover, the findings in this study should also facilitate future comparative genomic investigations between odonates and more modern insect groups, including model organisms like Apis mellifera, Drosophila melanogaster and Tribolium castaneum.

\section{Methods}

\section{Data collection and sample preparation}

One adult male I. elegans was collected from Alphen aan den Rijn in the Netherlands, on the $3^{\text {rd }}$ of August 2011. The individual was immediately euthanized in $\mathrm{EtOH}$ $(<10 \mathrm{sec})$ upon capture. The head, thorax and abdomen were separately crushed and stored in RNA later and from each of the three tissue types RNA was extracted. The tissue was homogenized using a bullet blender. Total RNA was extracted using an RNeasy kit (Qiagen) using the standardized instructions from the manufacturer. An aliquot of the extracts was used to quantify RNA using a RNA nano chip. mRNA was extracted, 
fragmented, converted to cDNA and fitted with adapters using standard protocols at the LGTC (Leiden Genome Technology Center, Netherlands). The libraries were PCR amplified for 16 cycles $(10 \mu \mathrm{l}$ cDNA prep, $10 \mu \mathrm{l}$ Phusion hot start buffer $7.5 \mathrm{mM} \mathrm{MgCl} 2,1 \mu \mathrm{l} 10 \mathrm{mM}$ dNTP's, $1 \mu \mathrm{l} \mathrm{P1,} 1 \mu \mathrm{l} \mathrm{P2,} 10 \mu \mathrm{l}$ DNA, $0.5 \mu \mathrm{l}$ Phusion, 20 $\mu \mathrm{l}$ water, $1 \mu \mathrm{l}$ USER; $30 \mathrm{~min} 37^{\circ} \mathrm{C}, 45 \mathrm{~min} 98^{\circ} \mathrm{C}, 10 \mathrm{~min}$ $98^{\circ} \mathrm{C}, 30 \mathrm{~min} 60^{\circ} \mathrm{C}$ for 15 cycles and $30 \mathrm{~min} 72^{\circ} \mathrm{C}$ ). Sequencing was performed in November 2011 with an Illumina HiSeq 2000 at LGTC using paired-end reads with an insert size of $280 \mathrm{bp}$ and an adapter length of $60 \mathrm{bp}$.

RNA sequence data has been deposited in the National Center for Biotechnology Information (NCBI) database under Ischnura elegans BioProject: PRJNA245854, which contains links and access to insect sampling data through the BioSample link: SAMN02741069 and the Sequence Read Archive: SRR1265958.

\section{Data processing and de novo transcriptome assembly}

The raw sequencing reads were trimmed by removing adapter sequences. Low quality sequences with an average quality score of less than 20 were removed using Nesoni clip version 0.109. Subsequently, reads with a length of less than $24 \mathrm{bp}$ were also discarded and the remaining reads were used for the assembly. The trimmed reads from head, thorax and abdomen were de novo assembled using Trinity version trinityrnaseq_r2012-06-08 $[28,29]$. Trinity generates transcriptome assemblies from short read sequences using the de Bruijn graph algorithm. The parameters selected to run Trinity were all default parameters (kmer length $=25$-mers) except min_kmer_cov which was set to 2 .

\section{Assembly quality assessment}

In order to assess the quality of the assembly, the Alignment Visualization and Quality Assessment application within Trinity software was used. This maps the reads back to the assembled transcripts using the bowtie aligner. The mapping results were visualized using Integrated Genomics Viewer version 2.3.2 (IGV) [30].

To improve the quality of the assembly, duplicates were removed and an internal quality check was performed. To remove duplicates from the assembly, clustering was performed using CD-HIT-EST at 95\% sequence similarity [34]. The application genome coverage bed within BED Tools version 2.17.0 was used to calculate the read coverage at each base. The transcripts with a mean coverage per base of less than five were removed from the assembly, because of the increased likelihood that these had been misassembled. The assembled transcripts were also screened for repetitive elements and rRNA using RepeatMasker version 4.0.1 using the default mode [35]. RepeatMasker was run with rmblastn version 2.2.27+ on RepBase update 20130422 and RM database version
20130422. The sequence completeness of the assembly was estimated with CEGMA software [36] and TargetIdentifier [37]. CEGMA version 2.4.010312 was used to evaluate the completeness of a transcriptome assembly by estimating the presence and completeness of 248 ultraconserved eukaryotic genes. It uses profile-hidden Markov model to ensure reliability of gene structure. Default parameters were used to run CEGMA. TargetIdentifier identifies the full-length transcripts using the BLASTX alignment as a guide to identify the protein coding regions and potential start and stop codons. The parameters that were used to run BLASTX are -v 1 -b 1 1E-5 on NCBI non-redundant protein database. Likely coding regions (Open Reading Frame) in the transcripts were identified using Transdecoder, which is an application within the Trinity software version trinityrnaseq_r2013_08_14.

\section{Functional annotation of transcripts}

High quality transcripts were annotated with the BLAST2GO [38], a comprehensive suit designed for the functional annotation and analysis of gene and protein sequences. The sequence homology search was conducted with BLASTX against the NCBI non-redundant (nr) protein database version $13^{\text {th }}$ November 2013 using an evalue cutoff of $1 \mathrm{E}-5$. The conserved motifs/domains were identified using InterProScan on the six possible translational frames of each transcript. The transcripts were functionally annotated according to the Gene Ontology nomenclature. InterProScan ID's were also mapped to GO terms and were merged with blast derived GO annotations in order to obtain one fully integrated annotation result. The GO annotations were further refined into biological processes, cellular components and molecular functional annotations. A GO_Slim reduction was performed on GO terms to obtain more precise GO definitions. Default settings were used to perform BLAST2GO, GO_Slim, GO Term enrichment and InterProScan analysis.

\section{Abundance estimation and differential expression}

An abundance estimation of the transcriptome assembly was obtained with the RSEM version 1.2.7 [41], separately for the three sets of filtered reads from the head, thorax and abdomen. RSEM is a package used to estimate the gene and isoform expression levels from RNA sequence data. RSEM was run using the default parameters except the seed-length, which was set to 24 , while calculating the expression. The relative measure of transcript abundance was TPM (Transcripts Per Million) and FPKM (Fragments Per Kilobase of transcript per Million mapped reads).

Differentially expressed transcripts were identified using edgeR Bioconductor [76]. EdgeR uses a negative binomial distribution method for differential expression analysis. We used edgeR through 'Identification and 
analysis of differentially expressed genes and transcripts' application with Trinity software versiontrinityrnaseq r20140413 at default settings.

\section{Interspecific comparisons}

The quality trimmed reads were mapped to the D. melanogaster transcriptome (downloaded on October $20^{\text {th }}$ 2013 from the Ensembl database [Ensembl:BDGP5]) [27]. In addition, we mapped the paired-end reads from L. fulva (downloaded from Baylor College of Medicine Human Genome Sequencing Center ftp site under the I5K project [BCM-HGSC:I5K]) [25] and the single end reads from $E$. Hageni (downloaded from NCBI [NCBI:SRR649536] submitted by BioProject number PRJNA185185 ID:185185) [26] to the I. elegans transcriptome. All mapping was performed using Bowtie2 with default parameters accompanied by Samtools for format conversions and for summarizing the mapping statistics [77,78]. We considered only those transcripts as mapped to which more than three reads were aligned.

All the computations were performed on resources provided by SNIC through Uppsala Multidisciplinary Center for Advanced Computational Science (UPPNEX) under Project b2013227 [79].

\section{Additional files}

Additional file 1: Table S1. Summary of repeats identified in I. elegans. Table S2. Comparative assembly statistics before and after filtering. Table S3. List of 30 most expressed genes from D. melanogaster that expressed in I. elegans. Table S4. List of 30 most expressed genes from I. elegans that expressed in E. hageni and L. fulva. Table S5. List of 20 most expressed genes in head of I. elegans. Table S6. List of 20 most expressed genes in thorax of I. elegans. Table S7. List of 20 most expressed genes in abdomen of I. elegans. Table S8. List of 20 differential expressed genes in head of I. elegans. Table S9. List of 20 most differential expressed genes in thorax of I. elegans. Table S10. List of 20 most differentially expressed genes in abdomen of I. elegans. Table S11. Expression and similarity statistics of different type of opsins expressed in I. elegans. Table S12. Details of locus similarity and expression levels of pigmentation enzymes and regulatory elements of I. elegans. Table S13. Odorant-binding proteins, olfactory receptor, ionotropic receptors and gustatory receptors identified in I. elegans with their expression in three tissues. Table S14. Expression and similarity statistics of different type of Heat and cold shock proteins expressed in I. elegans. Table S15. Reports different types of TRPs identified in I. elegans with their expression in three tissues.

Additional file 2: Figure S1. Comparison between filtered (final) and un-filtered (initial) transcriptome of l.elegans. Figure S2. Gene Ontology annotation for biological process category I. elegans. Figure S3. Gene Ontology annotation for Cellular Component category I. elegans. Figure S4. Gene Ontology annotation for Molecular Function category I. elegans. Figure S5. Venn diagram deciphering the distribution of E. hageni (blue) and L. fulva (yellow) transcripts expressed in I. elegans. Figure S6. Enriched $\mathrm{GO}$ term distribution observed in head of I. elegans after reducing to most specific GO terms. Figure S7. Enriched GO term distribution observed in thorax of I. elegans. Figure S8. Enriched GO term distribution observed in abdomen of I. elegans after reducing to most specific $\mathrm{GO}$ terms.

\section{Competing interests}

The authors declared that they have no competing interest.
Authors' contributions

$\mathrm{PC}, \mathrm{BH}, \mathrm{ES}, \mathrm{KK}, \mathrm{PK}$ and $\mathrm{MW}$ designed the research. $\mathrm{PC}, \mathrm{BH}, \mathrm{KK}$ and $\mathrm{MW}$ performed research. KK contributed biological material. PC, MW and BH wrote the manuscript. All authors read and approved the final manuscript.

\section{Acknowledgements}

We would like to thank Phill Watts, Lesley Lancaster, Miriam Henze and Seth Bybee for comments on earlier versions of this manuscript, Martin Dahlö from the SciLifeLab and UPPNEX (project number b2013227), the national resource for Next Generation Sequencing in Sweden, for support. This study was supported by grants from the Swedish Research Council (to MW; grant no.: 2012-3996), the Crafoord Foundation (to BH, MW) and the Erik Philip-Sörensens Stiftelse (EPSS) (to BH, EIS).

\section{Author details}

'Department of Biology, Lund University, Sölvegatan 37, SE-22362 Lund, Sweden. ${ }^{2}$ Animal Ecology, Department of Ecological Science, VU University Amsterdam, De Boelelaan 1085, 1081 HV Amsterdam, The Netherlands. ${ }^{3}$ Department of Human and Clinical Genetics, Leiden University Medical Center, PO Box 9600, 2300 RC Leiden, The Netherlands.

Received: 6 March 2014 Accepted: 9 September 2014 Published: 22 September 2014

\section{References}

1. Bybee S, Johnson KK, Gering E, Whiting M, Crandall K: All the better to see you with: a review of odonate color vision with transcriptomic insight into the odonate eye. Organisms Diversity \& Evolution 2012, 12(3):241-250.

2. Warrant E, Nilsson D-E: Invertebrate vision. Cambridge: Cambridge University Press; 2006.

3. Corbet PS: Dragonflies: behavior and ecology of Odonata. Essex, UK: Harley Books; 1999

4. Samways MJ: Dragonflies as focal organisms in contemporary conservation biology. In Dragonflies and Damselflies. Edited by Córdoba-Aguilar A. Oxford: Oxford University Press; 2008.

5. Wellenreuther $M$, Tynkkynen $K$, Svensson El: Simulating range expansion: male species recognition and loss of premating isolation in damselflies. Evolution 2010, 64(1):242-252

6. Svensson E, Abbott J, Gosden T, Coreau A: Female polymorphisms, sexual conflict and limits to speciation processes in animals. Evol Ecol 2009, 23:93-108.

7. Svensson El, Waller JT: Ecology and Sexual Selection: Evolution of Wing Pigmentation in Calopterygid Damselflies in Relation to Latitude, Sexual Dimorphism, and Speciation. Am Nat 2013, 182(5):E174-E195.

8. Bybee SM, Ogden TH, Branham MA, Whiting MF: Molecules, morphology and fossils: a comprehensive approach to odonate phylogeny and the evolution of the odonate wing. Cladistics 2008, 34(4):477-514.

9. Shanku AG, McPeek MA, Kern AD: Functional Annotation and Comparative Analysis of a Zygopteran Transcriptome. G3 2013, 3(4):763-770.

10. Andrés $J A$, Cordero $A$ : The inheritance of female colour morphs in the damselfly Ceriagrion tenellum (Odonata, Coenagrionidae). Heredity 1999, 82(3):328-335

11. Gosden T, Svensson E: Density-dependent male mating harassment, female resistance, and male mimicry. Am Nat 2009, 173:709-721.

12. Sánchez-Guillén RA, Hammers M, Hansson B, Gossum HV, Cordero-Rivera A, Mendoza DIG, Wellenreuther M: Ontogenetic shifts in male mating preference and morph-specific polyandry in a female colour polymorphic insect. BMC Evol Biol 2013, 13(116):1-11.

13. Svensson El, Abbott J, Hardling R: Female polymorphism, frequency dependence, and rapid evolutionary dynamics in natural populations. Am Nat 2005, 165:567-576.

14. Miller PL: Sperm competition in Ischnura elegans (Vander Linden) (Zygoptera: Coenagrionidae). Odonatologica 1987, 16:201-207.

15. Cooper G, Miller PL, Holland PWH: Molecular genetic analysis of sperm competition in the damselfly Ischnura elegans (Vander Linden). Proc $R$ Soc Ser B 1996, 263:1343-1349.

16. Sánchez-Guillén RA, Córdoba-Aguilar A, Cordero-Rivera AS, Wellenreuther M: Genetic divergence predicts reproductive isolation in damselflies. J Evol Biol 2013, 7(1):76-87. 
17. Sánchez-Guillén RA, Wellenreuther M, Cordero-Rivera AS: Strong asymmetry in the relative strengths of prezygotic and postzygotic barriers between two damselfly sister species. Evolution 2012, 66(3):690-707.

18. Paulson D: Dragonflies and damselflies of the West. Princeton University Press: Princeton; 2009.

19. Fincke MO, Jödicke R, Paulson DR, Schultz TD: The evolution and frequency of female color morphs in Holarctic Odonata: why are malelike females typically the minority? Int J Odonatol 2005, 8(2):183-212.

20. Cordero A: The inheritance of female polymorphism in the damselfly Ischnura graellsii (Rambur) (Odonata: Coenagrionidae). Heredity 1990, 64:341-346.

21. Sánchez-Guillén RA, Gossum HV, Rivera AC: Hybridization and the inheritance of female colour polymorphism in two ischnurid damselflies (Odonata: Coenagrionidae). Biol J Linn Soc 2005, 85(4):471-481.

22. Gossum HV, Bots J, Heusden JV, Hammers M, Huyghe K, Morehouse Nl: Reflectance spectra and mating patterns support intraspecific mimicry in the colour polymorphic damselflylschnura elegans. Evol Ecol 2011, 25(1):139-154

23. Askew RR: The dragonflies of Europe. Colchester, UK: B H \& A Harley Ltd; 2004.

24. Wellenreuther M, Sánchez-Guillén RA, Cordero-Rivera A, Svensson El Hansson B: Environmental and climatic determinants of molecular diversity and genetic population structure in a coenagrionid damselfly. PLoS One 2011, 6(6):e20440

25. Ladona fulva RNA sequence data download website. ftp://ftp.hgsc.bcm.edu/ I5K-pilot/Scarce_Chaser/RNA_sequence/.

26. Enallagma hageni RNA sequence data download website. ftp://ftp-trace.ncbi. nlm.nih.gov/sra/sra-instant/reads/ByRun/sra/SRR/SRR649/SRR649536.

27. Drosophila melanogaster transcriptome data download website. ftp://ftp. ensembl.org/pub/release-75/fasta/drosophila_melanogaster/cdna/.

28. Haas BJ, Papanicolaou A, Yassour M, Grabherr M, Blood PD, Bowden J, Couger MB, Eccles D, Li B, Lieber M, MacManes MD, Ott M, Orvis J, Pochet N, Strozzi F, Weeks N, Westerman R, William T, Dewey CN, Henschel R, LeDuc RD, Friedman N, Regev A: De novo transcript sequence reconstruction from RNA-seq using the Trinity platform for reference generation and analysis. Nat Protocols 2013, 8(8):1494-1512.

29. Grabherr MG, Haas BJ, Yassour M, Levin JZ, Thompson DA, Amit I, Adiconis X, Fan L, Raychowdhury R, Zeng Q, Chen Z, Mauceli E, Hacohen N, Gnirke A, Rhind N, Palma FD, Birren BW, Nusbaum C, Lindblad-Toh K, Friedman N, Regev A: Full-length transcriptome assembly from RNA-Seq data without a reference genome. Nat Biotechnol 2011, 29(7):644-652

30. Thorvaldsdottir H, Robinson JT, Mesirov JP: Integrative Genomics Viewer (IGV): high-performance genomics data visualization and exploration. Brief Bioinform 2013, 14(2):178-192.

31. Pallavicini A, Canapa A, Barucca M, Alfoldi J, Biscotti M, Buonocore F, De Moro G, Di Palma F, Fausto A, Forconi M, Gerdol M, Makapedua DM, Turner-Meier J, Olmo E, Scapigliati G: Analysis of the transcriptome of the Indonesian coelacanth Latimeria menadoensis. BMC Genomics 2013, 14(1):538.

32. Liu S, Zhang Y, Zhou Z, Waldbieser G, Sun F, Lu J, Zhang J, Jiang Y, Zhang H, Wang X, Rajendran KV, Khoo L, Kucuktas H, Peatman E, Liu Z: Efficient assembly and annotation of the transcriptome of catfish by RNA-Seq analysis of a doubled haploid homozygote. BMC Genomics 2012, 13:595.

33. Miller $H$, Biggs $P$, Voelckel $C$, Nelson N: De novo sequence assembly and characterisation of a partial transcriptome for an evolutionarily distinct reptile, the tuatara (Sphenodon punctatus). BMC Genomics 2012 13(1):439.

34. Li W, Godzik A: Cd-hit: a fast program for clustering and comparing large sets of protein or nucleotide sequences. Bioinformatics 2006, 22(13):1658-1659.

35. RepeatMasker Open-3.0. http://www.repeatmasker.org/.

36. Parra G, Bradnam K, Korf I: CEGMA: a pipeline to accurately annotate core genes in eukaryotic genomes. Bioinformatics 2007, 23(9):1061-1067.

37. Min XJ, Butler G, Storms R, Tsang A: Targetldentifier: a webserver for identifying full-length cDNAs from EST sequences. Nucleic Acids Res 2005, 33(suppl 2):W669-W672.

38. Conesa A, Gotz S, Garcia-Gomez JM, Terol J, Talon M, Robles M: Blast2GO: a universal tool for annotation, visualization and analysis in functional genomics research. Bioinformatics 2005, 21(18):3674-3676.

39. Trautwein MD, Wiegmann BM, Beutel R, Kjer KM, Yeates DK: Advances in insect phylogeny at the dawn of the postgenomic era. Annu Rev Entomol 2012, 57:449-468
40. Meusemann K, von Reumont BM, Simon S, Roeding F, Strauss S, Kück P, Ebersberger I, Walzl M, Pass G, Breuers S, Achter V, Haeseler AV, Burmester T, Hadrys H, Wägele JW, Misof B: A phylogenomic approach to resolve the arthropod tree of life. Mol Biol Evol 2010, 27(11):2451-2464.

41. Li B, Dewey C: RSEM: accurate transcript quantification from RNA-Seq data with or without a reference genome. BMC Bioinformatics 2011, 12(1):323.

42. Altincicek B, Vilcinskas A: Identification of immune-related genes from an apterygote insect, the firebrat Thermobia domestica. Insect Biochem Mol Biol 2007, 37(7):726-731.

43. Pomes A, Chapman MD, Vailes LD, Blundell TL, Dhanaraj V: Cockroach allergen Bla g 2: structure, function, and implications for allergic sensitization. Am J Respir Crit Care Med 2002, 165(3):391-397.

44. Bluthgen N, Brand K, Cajavec B, Swat M, Herzel H, Beule D: Biological profiling of gene groups utilizing Gene Ontology. Genome Inform 2005, 16:106-115.

45. Meinertzhagen IA, Menzel R, Kahle G: The identification of spectral receptor types in the retina and lamina of the dragonfly Sympetrum rubicundulum. J Comp Physiol 1983, 151(3):295-310.

46. Wittkopp PJ, Carroll SB, Kopp A: Evolution in black and white: genetic control of pigment patterns in Drosophila. Trends Genet 2003, 19(9):495-504.

47. Wittkopp PJ, Beldade P: Development and evolution of insect pigmentation: Genetic mechanisms and the potential consequences of pleiotropy. Semin Cell Dev Biol 2009, 20(1):65-71.

48. True JR: Insect melanism: the molecules matter. Trends Ecol Evol (Personal Edition) 2003, 18(12):640-647.

49. Siva-Jothy MT: A mechanistic link between parasite resistance and expression of a sexually selected trait in a damselfly. Proc $R$ Soc Lond $B$ Biol Sci 2000, 267(1461):2523-2527.

50. Svensson El, Eroukhmanoff F, Karlsson K, Runemark A, Brodin A: A role for learning in population divergence of mate preferences. Evolution 2010, 64(11):3101-3113.

51. Neckameyer WS: Dopamine and mushroom bodies in Drosophila: experience-dependent and -independent aspects of sexual behavior. Learn Mem 1998, 5(1-2):157-165.

52. Neckameyer WS: Dopamine modulates female sexual receptivity in Drosophila melanogaster. J Neurogenet 1998, 12(2):101-114.

53. Wicker-Thomas C, Hamann M: Interaction of dopamine, female pheromones, locomotion and sex behavior in Drosophila melanogaster. J Insect Physiol 2008, 54(10-11):1423-1431.

54. Liu T, Dartevelle L, Yuan C, Wei H, Wang Y, Ferveur JF, Guo A: Increased dopamine level enhances male-male courtship in Drosophila. J Neurosc 2008, 28(21):5539-5546.

55. Keleman K, Vrontou E, Kruttner S, Yu JY, Kurtovic-Kozaric A, Dickson BJ: Dopamine neurons modulate pheromone responses in Drosophila courtship learning. Nature 2012, 489(7414):145-149.

56. Han Q, Fang J, Ding H, Johnson JK, Christensen BM, Li J: Identification of Drosophila melanogaster yellow-f and yellow- $\mathrm{f} 2$ proteins as dopachromeconversion enzymes. Biochem J 2002, 368(Pt 1):333-340.

57. Wright TRF, Bewley GC, Sherald AF: The genetics of dopa decarboxylase in Drosophila melanogaster. II. Isolation and characterization of dopadecarboxylase-deficient mutants and their relationship to the alphamethyl-dopa-hypersensitive mutants. Genetics 1976, 84(2):287-310.

58. Yu H-S, Shen Y-H, Yuan G-X, Hu Y-G, Xu H-E, Xiang Z-H, Zhang Z: Evidence of selection at melanin synthesis pathway loci during silkworm domestication. Mol Biol Evol 2011, 28(6):1785-1799.

59. Asano T, Takebuchi K: Identification of the gene encoding prophenoloxidase A(3) in the fruitfly, Drosophila melanogaster. Insect Mol Biol 2009, 18(2):223-232.

60. Asada N, Yokoyama G, Kawamoto N, Norioka S, Hatta T: Prophenol oxidase A3 in Drosophila melanogaster: activation and the PCR-based CDNA sequence. Biochem Genet 2003, 41(5-6):151-163.

61. Reed RD, Nagy LM: Evolutionary redeployment of a biosynthetic module: expression of eye pigment genes vermilion, cinnabar, and white in butterfly wing development. Evol Dev 2005, 7(4):301-311.

62. Yamamoto M, Howells AJ, Ryall RL: The ommochrome biosynthetic pathway in Drosophila melanogaster: the head particulate phenoxazinone synthase and the developmental onset of xanthommatin synthesis. Biochem Genet 1976, 14(11-12):1077-1090.

63. Osanai-Futahashi M, Tatematsu K-i, Yamamoto K, Narukawa J, Uchino K, Kayukawa T, Shinoda T, Banno Y, Tamura T, Sezutsu H: Identification of the 
Bombyx Red Egg Gene Reveals Involvement of a Novel Transporter Family Gene in Late Steps of the Insect Ommochrome Biosynthesis Pathway. J Biol Chem 2012, 287(21):17706-17714.

64. Pao SS, Paulsen IT, Saier MH Jr: Major facilitator superfamily. Microbiol Mol Biol Rev 1998, 62(1):1-34.

65. Braasch I, Schartl M, Volff JN: Evolution of pigment synthesis pathways by gene and genome duplication in fish. BMC Evol Biol 2007, 7:74.

66. Meng Y, Katsuma S, Daimon T, Banno Y, Uchino K, Sezutsu H, Tamura T, Mita K, Shimada T: The silkworm mutant lemon (lemon lethal) is a potential insect model for human sepiapterin reductase deficiency. J Biol Chem 2009, 284(17):11698-11705.

67. Ziegler I: The pteridine pathway in zebrafish: regulation and specification during the determination of neural crest cell-fate. Pigment Cell Res 2003, 16(3):172-182

68. Hadrys H, Simon S, Kaune B, Schmitt O, Schöner A, Jakob W, Schierwater B. Isolation of Hox Cluster Genes from Insects Reveals an Accelerated Sequence Evolution Rate. PLoS One 2012, 7(6):e34682.

69. Bickel RD, Kopp A, Nuzhdin SV: Composite Effects of Polymorphisms near Multiple Regulatory Elements Create a Major-Effect QTL. PLoS Genet 2011, 7(1):e1001275

70. Rebora M, Salerno G, Piersanti S, Dell'otto A, Gaino E: Olfaction in dragonflies: electrophysiological evidence. J Insect Physiol 2012, 58(2):270-277.

71. Piersanti S, Frati F, Conti E, Gaino E, Rebora M, Salerno G: First evidence of the use of olfaction in Odonata behaviour. J Insect Physiol 2014, 62:26-31.

72. Hallem EA, Dahanukar A, Carlson JR: Insect odor and taste receptors. Annu Rev Entomol 2006, 51:113-135.

73. May ML: Thermoregulation and Adaptation to Temperature in Dragonflies (Odonata: Anisoptera). Ecol Monogr 1976, 46(1):1-32.

74. May ML: Thermoregulation and Reproductive Activity in Tropical Dragonflies of the Genus Micrathyria. Ecology 1977, 58(4):787-798

75. May ML: Energy-Metabolism of Dragonflies (Odonata, Anisoptera) at Rest and During Endothermic Warm-Up. J Exp Biol 1979, 83:79-94.

76. Robinson MD, McCarthy DJ, Smyth GK: Edger: a bioconducter package for differential expression analysis of digital gene expression data. Bioinformatics 2010, 26(1):139-140.

77. Langmead B, Salzberg SL: Fast gapped-read alignment with Bowtie 2. Nat Meth 2012, 9(4):357-359.

78. Li H, Handsaker B, Wysoker A, Fennell T, Ruan J, Homer N, Marth G, Abecasis G, Durbin R: The Sequence Alignment/Map format and SAMtools. Bioinformatics 2009, 25(16):2078-2079.

79. Lampa S, Dahlo M, Olason P, Hagberg J, Spjuth O: Lessons learned from implementing a national infrastructure in Sweden for storage and analysis of next-generation sequencing data. Giga Sci 2013, 2(1):9.

doi:10.1186/1471-2164-15-808

Cite this article as: Chauhan et al:: De novo transcriptome of Ischnura elegans provides insights into sensory biology, colour and vision genes. BMC Genomics 2014 15:808.

\section{Submit your next manuscript to BioMed Central and take full advantage of:}

- Convenient online submission

- Thorough peer review

- No space constraints or color figure charges

- Immediate publication on acceptance

- Inclusion in PubMed, CAS, Scopus and Google Scholar

- Research which is freely available for redistribution

Submit your manuscript at www.biomedcentral.com/submit
C BioMed Central 